\title{
Ermüdungssicherheit von Brücken - Teil 2: Nachweis basierend auf den Messwerten des Monitoring-Projekts „Bahnbrücke Eglisau“
}

Bei der genieteten Rheinbrücke in Eglisau wurde über einen Zeitraum von einem Jahr ein Monitoring durchgeführt. Die mittels Rainflow-Analyse ausgewerteten Messwerte dienten als Grundlage für den Nachweis der Ermüdungssicherheit. Die Messquerschnitte sind in der Regel nicht identisch mit den Nachweisquerschnitten, weshalb die gemessenen Dehnungen bzw. Spannungen in die für den Nachweis maßgebende Nietlage des Nachweisquerschnittes umgerechnet wurden. Die hierfür erforderlichen Umrechnungsfaktoren wurden rechnerisch am statischen Modell ermittelt. In einem ersten Schritt wurde die Dauerfestigkeit für die ermüdungsbeanspruchten Bauteile untersucht. Für die Bauteile mit ungenügender Dauerfestigkeit wurde anschließend eine Schadensakkumulationsberechnung nach Palmgren-Miner auf Basis der für genietete Konstruktionsdetails geltenden Wöhlerkurven durchgeführt. Basierend auf den Messwerten aus dem Monitoring konnte schließlich für die Nietkonstruktion eine genügende Ermüdungssicherheit und für das maßgebende Bauteil eine weitere Nutzungsdauer von mindestens 50 Jahren nachgewiesen werden.

Fatigue safety of riveted bridges - Part 2: Verification based on the monitoring data of the project „Railway Bridge at Eglisau“. Long term monitoring over one year has been conducted on the riveted Railway Bridge over the Rhine at Eglisau. Measured values were exploited by rainflow analysis and served as the basis for the verification of fatigue safety. As the locations of measurements are generally not identical with the cross sections of verification, measured strains respectively stresses, were extrapolated to the relevant verification cross section by means of factors that were obtained by structural analysis. Using these values, all fatigue relevant structural details were first verified with respect to the fatigue limit. Then, damage accumulation calculation according to the Palmgren-Miner rule and based on Wöhler curves for riveted details was performed for those structural details where the fatigue limit check was not fulfilled. Sufficient fatigue safety could finally be verified for the whole riveted structure and an additional service life of at least 50 years for the most fatigue relevant structural element. baren, modularen Messkonzept erfasst werden [1]. Ziel des Projektes war es, basierend auf den Monitoring-Daten das tatsächliche Tragverhalten ausreichend genau beurteilen zu können.

Die Projektabwicklung erfolgte in vier Phasen über insgesamt zwei Jahre. Die letzte Projektphase beinhaltete die Erfassung der Monitoring-Daten über ein Jahr sowie die Nachweisführung für die Trag- und Ermüdungssicherheit. Nachfolgend wird der auf Monitoring-Daten basierende Nachweis der Ermüdungssicherheit erläutert.

\section{Beschreibung der Brücke}

Die Rheinbrücke in Eglisau (Schweiz) wurde 1895 bis 1897 für einen eingleisigen Bahnverkehr gebaut. Im zentralen Bereich des $457 \mathrm{~m}$ langen Bauwerkes wird der Rhein mit einer genieteten Stahlfachwerkkonstruktion über $90 \mathrm{~m}$ frei tragend überspannt. Nördlich und südlich der $9 \mathrm{~m}$ hohen Fachwerkkonstruktion schließen sich die beiden gemauerten Vorlandbrü-

\section{Einleitung}

Die steigenden Anforderungen des modernen Bahnverkehrs stellen die Eigentümerschaften von Bahnbrücken zunehmend vor die Aufgabe, ihren Bauwerksbestand hinsichtlich der Sicherheit und Restnutzungsdauer zu beurteilen. Es sind deshalb zuverlässige Aussagen bezüglich der Tragsicherheit, Gebrauchstauglichkeit und Ermüdungssicherheit notwendig. Im Rahmen des Monitoring-Projektes „Bahnbrücke Eglisau“ konnten die zur Beurteilung von Sicherheit und Restnutzungsdauer erforderlichen Informationen mit einem leicht anpass-

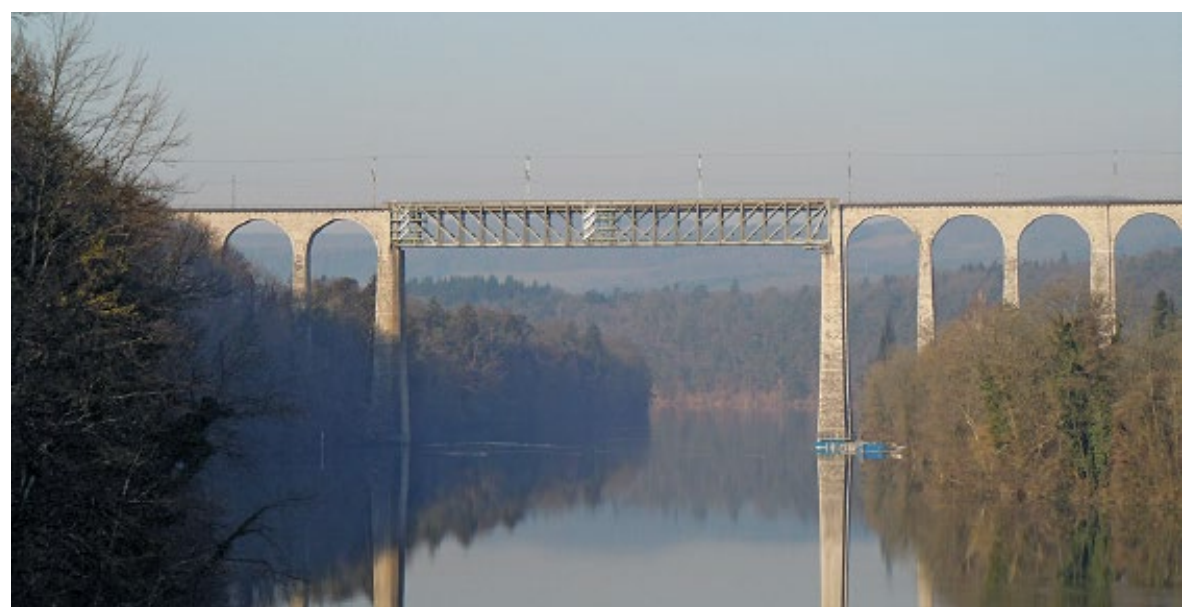

Bild 1. Bahnbrücke über den Rhein bei Eglisau, Schweiz (Foto: privat) Fig. 1. Railway bridge over the Rhine at Eglisau, Switzerland 
cken an, deren Pfeiler im Auflagerbereich bis zu $50 \mathrm{~m}$ hoch sind (Bild 1).

Im Jahr 1921 wurde auf der Seite mit dem beweglichen Lager eine Verspannvorrichtung eingebaut, die mittels Hebelwirkung je 1000 kN Druckkraft in die Fachwerkuntergurte einleitet und so den festgestellten horizontalen Verschiebungen der Uferpfeiler entgegenwirkt. Basierend auf einer im Jahr 1980 durchgeführten statischen Überprüfung wurden 1982/83 die ursprüngliche Fahrbahn aus Zores-Eisen durch einen Schottertrog ersetzt sowie mehrere Stabanschlüsse verstärkt. Der Korrosionsschutz der Stahlkonstruktion wurde zudem vollständig erneuert.

\section{Modellbildung und Tragwerks- analyse}

In der ersten Projektphase wurde parallel zur Optimierung des Messkonzeptes das Tragwerk mit einem dreidimensionalen Stabmodell erfasst, welches zur Schnittkraftermittlung im Rahmen des Tragsicherheitsnachweises verwendet wurde (Bild 2).

Zur Funktionskontrolle der Messeinrichtung wurden vor Beginn der Langzeitmessung im September 2010 Belastungsversuche mit Lokomotiven durchgeführt. Ein Vergleich der Messergebnisse mit den Rechenwerten diente der Optimierung des Messkonzepts, indem bei den bezüglich Ermüdung wichtigsten Querschnitten zusätzliche Messstellen angeordnet wurden.

Die Anordnung der Fahrbahn (bestehend aus Schottertrog, Zwischenquerträger, Längsträger, Querträger) etwa 1,5 $\mathrm{m}$ unterhalb des Hauptfachwerk-Obergurtes hat zur Folge, dass die Fahrbahn auch als Obergurt mitträgt und entsprechend Druckkräfte abgetragen werden. Aufgrund dieser Tragfunktion erfolgte die Modellierung der Fahrbahn auf der Höhe OK Querträger, da hier der obere Windverband angeordnet ist und dementsprechend die horizontalen Lasten mehrheitlich auf dieser Höhe seitlich in die Pfosten abgetragen werden. Die Längsträger und der Schottertrog, welcher mit vier fiktiven Trägern modelliert wird, werden entsprechend ihrer wirklichen Lage exzentrisch an die Querträger angeschlossen (Bild 3).

Beim Hauptfachwerk werden alle Knoten unter Berücksichtigung ex-
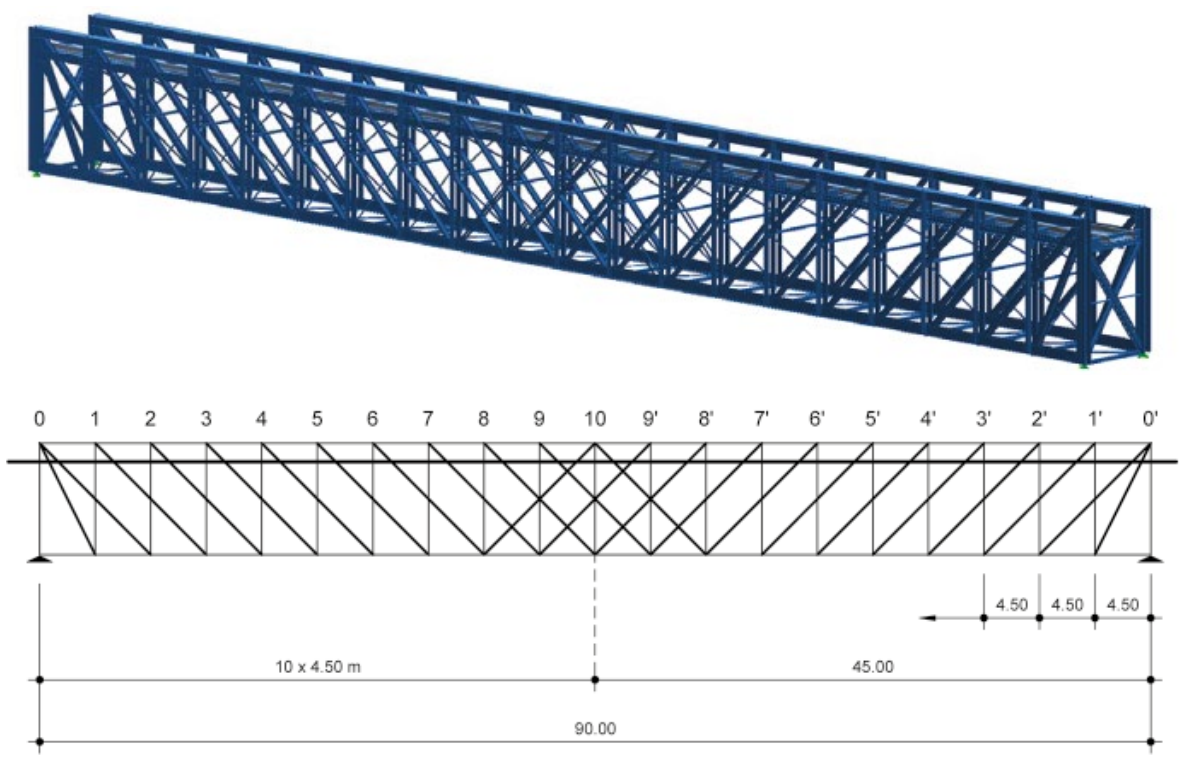

Bild 2. Dreidimensionales Modell für die Tragwerksanalyse und Abmessungen des Hauptträgers

Fig. 2. Three-dimensional model for the structural analysis and dimensions of the main girder

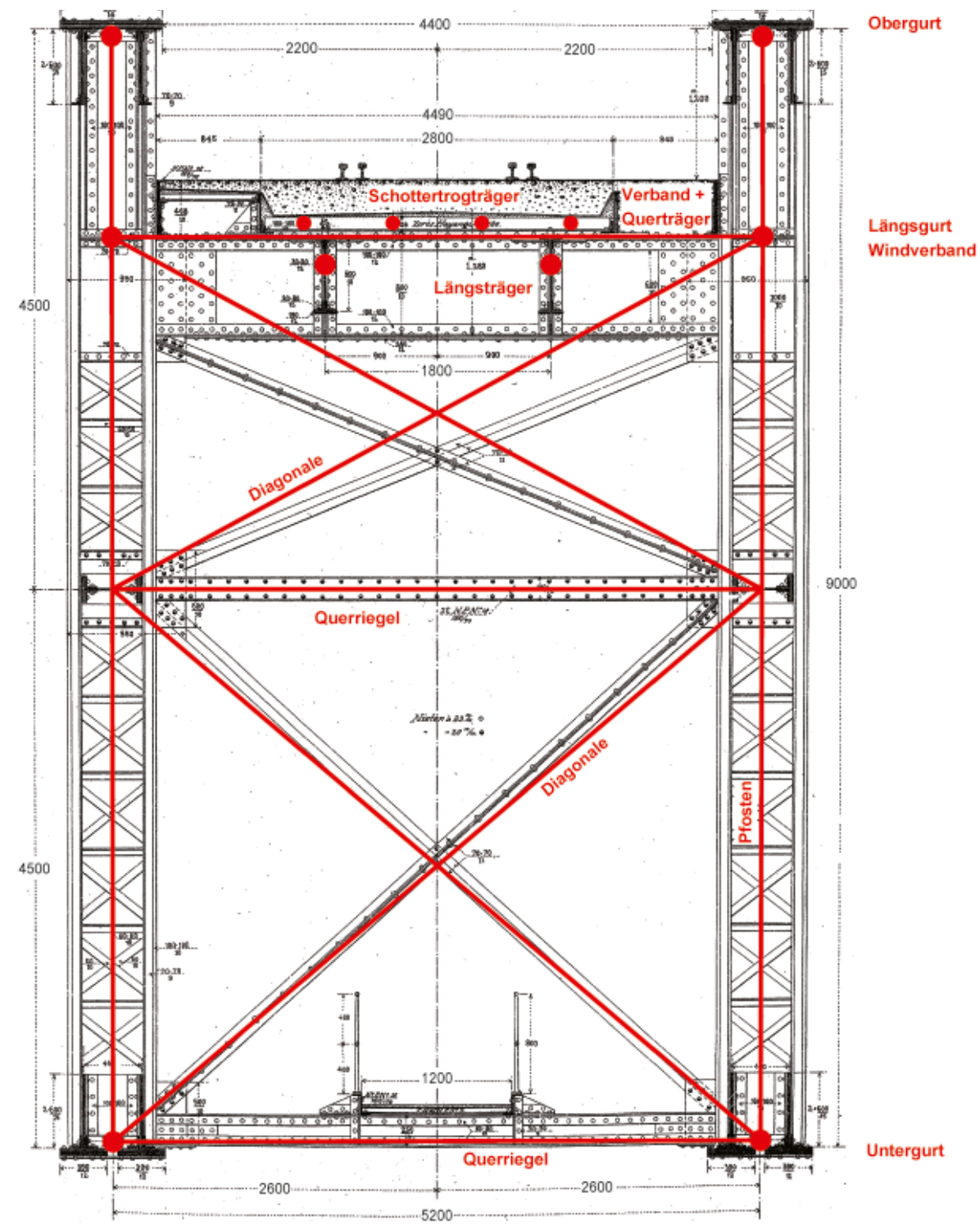

Bild 3. Querschnitt des Fachwerkträgers und Modellierung

Fig. 3. Cross section of the truss girder and modelling 
zentrischer Anschlüsse biegesteif modelliert. Die Füllstäbe des oberen und unteren Windverbandes sowie der Querverbände werden hingegen gelenkig angeschlossen.

Ein Vergleich der Messergebnisse aus dem Belastungsversuch mit den am Tragwerksmodell ermittelten rechnerischen Werten zeigte auf, dass das Tragverhalten mit dem statischen Modell ausreichend genau erfasst wird.

Mit dem Einbau des Schottertroges im Jahre 1982 wurde die Beanspruchung der Längsträger deutlich reduziert. Es war deshalb zu erwarten, dass der Fahrbahnbereich mit Schottertrog und Längsträger bezüglich aktueller Ermüdungsbeanspruchungen unkritisch sein dürfte.

\section{Ergebnisse des Langzeit-Monitorings}

Die zur Beurteilung der Ermüdungssicherheit relevanten Dehnmessstreifen wurden an folgenden Bauteilen montiert (vgl. Bild 4):

Feld 1: Diagonalen, Längsträger, Querträger, Pfosten

Feld 10: Untergurt

Im Bild 5 sind beispielhaft einzelne Querschnitte mit der Anordnung der Dehnmessstreifen dargestellt.

Die Messdaten jeder Zugsüberfahrt wurden gespeichert und können in Funktion der Zeit grafisch dargestellt werden. In Bild 6 sind beispielhaft für einzelne Messstellen die Messdaten für eine Güterzugüberfahrt festgehalten. Dargestellt sind die Dehnungen in Funktion der Zeit. Zu beachten ist, dass bei dieser grafischen Darstellung der Maßstab der Ordinate nicht konstant ist. Deutlich sichtbar sind bei Diagonale und Untergurt die geringen Einflüsse der einzelnen Achslasten, während beim Querträger vorerst ein kontinuierlicher Anstieg (globale Wirkung) und anschließend
Pfosten
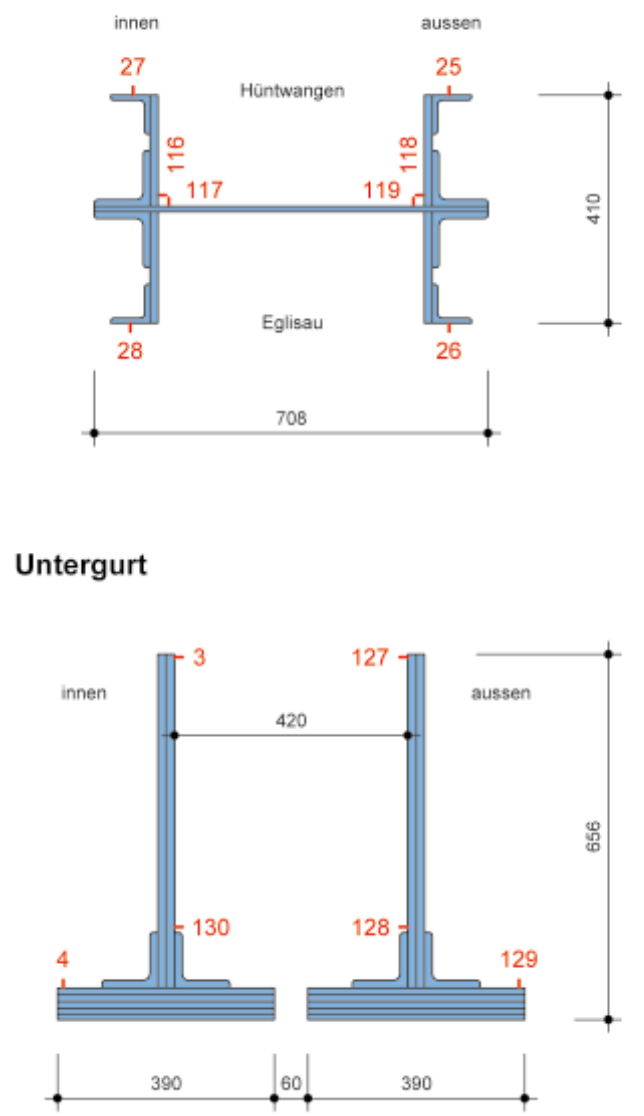

1. Diagonale

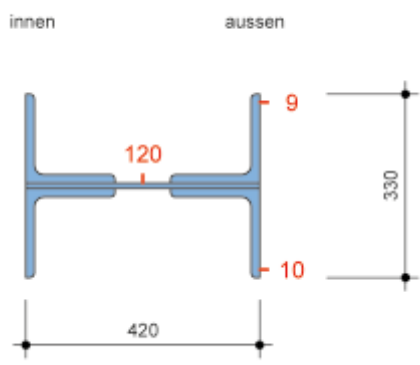

Querträger

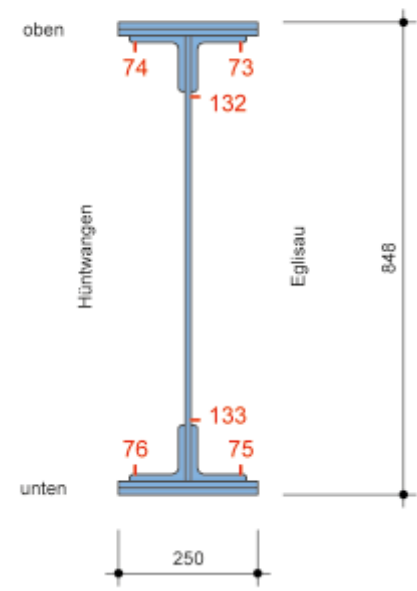

Bild 5. Anordnung der Dehnmessstreifen in den Querschnitten

Fig. 5. Arrangement of strain gauges in cross sections

das direkte Überfahren der Achsen mit prozentual starken Dehnungsänderungen (lokale Wirkung) unterschieden werden kann.

\section{Nachweis der Ermüdungssicherheit 5.1 Vorgehenskonzept}

Neben dem Aspekt der Zugänglichkeit wurde die Lage der DMS so gewählt, dass die Spannungsverteilung im Messquerschnitt möglichst einem elastischen Verhalten im Bruttoquerschnitt entspricht. Die Messstellen liegen deshalb möglichst weit entfernt zu Nietlöchern, um Dehnungskon-
Eglisau

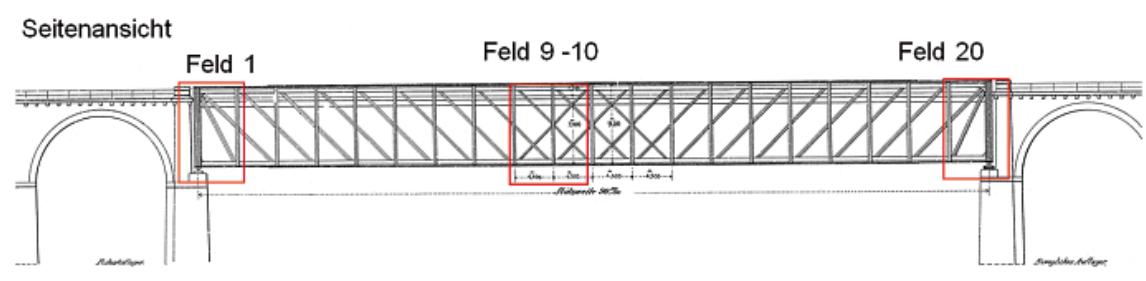

Bild 4. Lokalisierung der zur Beurteilung der Ermüdungssicherheit relevanten Dehnmessstreifen

Fig. 4. Localisation of strain gauges relevant for the fatigue safety verification zentrationen nahe der Niete zu vermeiden. Im Nachweisquerschnitt sind hingegen die Lagen der Nietlöcher ermüdungsrelevant, weshalb die gemessenen Dehnungen bzw. Spannungen für den Nachweis auf die maßgebende Nietlage im Nachweisquerschnitt umgerechnet werden mussten (vgl. Abschnitt 5.3). Für die Nachweisquerschnitte wurden in einer ersten Stufe der Dauerfestigkeitsnachweis und falls notwendig in einer zweiten Stufe der Ermüdungsnachweis mit der Schadensakkumulationstheorie nach Palmgren-Miner geführt.

\subsection{Messwerte}

Die Messwerte jeder einzelnen Zugüberfahrt wurden mittels der Rainflow-Analyse ausgewertet und gewählten Dehnungs- bzw. Spannungsstufen zugeordnet. Jeweils über eine Messdauer von einem Monat wurden die Messwerte aufsummiert und abgespeichert. Die entsprechenden Monatsauswertungen wurden als Tabellen sowie grafisch als Histogramme aufbereitet (Bild 7). 


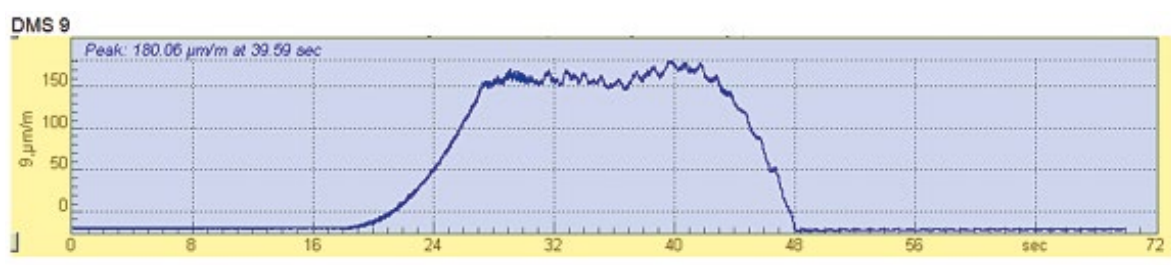

1. Diagonale

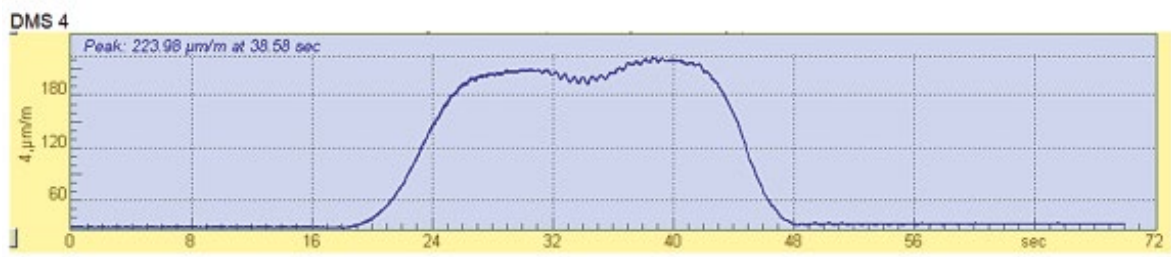

Untergurt in Feldmitte

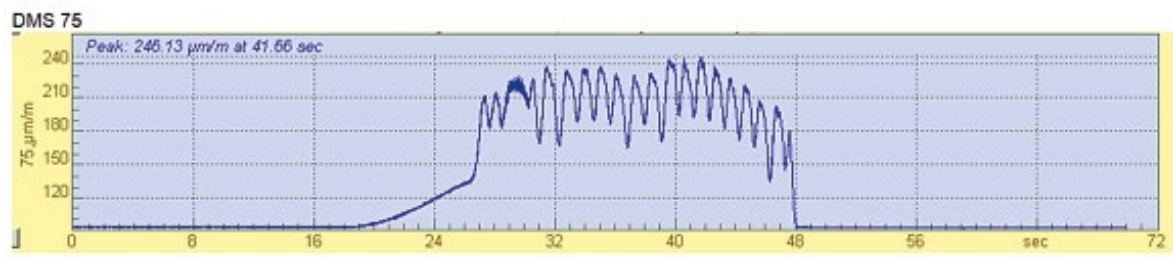

Quertrăger

Bild 6. Aufzeichnung einzelner DMS der 1. Diagonalen, des Untergurts in Feldmitte und des Querträgers

Fig. 6. Recorded data of a strain gauge on the 1st diagonal, the bottom chord at mid-span and the cross girder

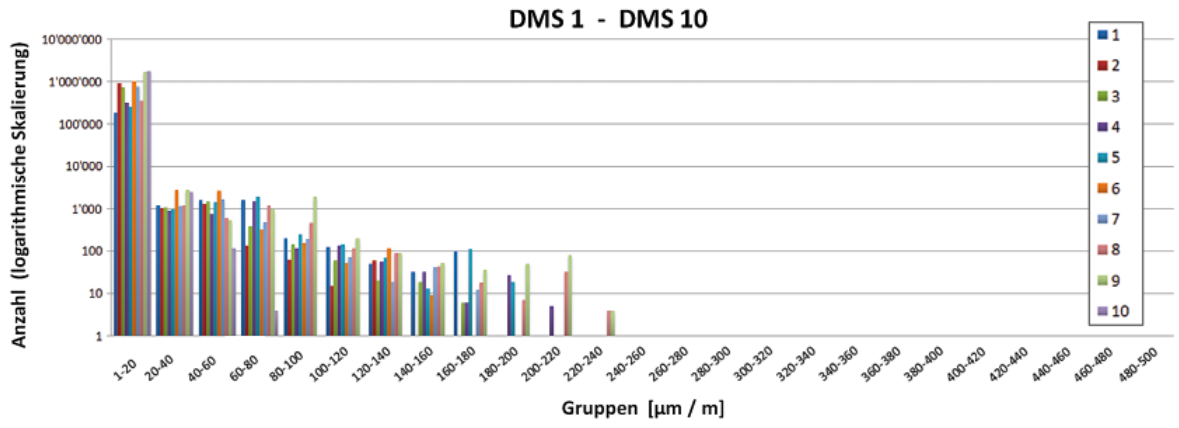

Bild 7. Histogramm einer Monatsauswertung

Fig. 7. Histogram of a monthly evaluation

\subsection{Umrechnung Messquerschnitt - Nachweisquerschnitt}

Die Umrechnung der Messwerte in die maßgebende Nietlage im Nachweisquerschnitt erfolgte mittels Umrechnungsfaktoren, die rechnerisch am dreidimensionalen Stabmodell anhand der Schnittkraftverteilung für eine zentrische Anordnung des Lastmodells D4 gemäß der Norm SIA 269/1 [2] ermittelt wurden. Lediglich für die Längsträger wurde der Umrechnungsfaktor mit gezielten Laststellungen für das maximale und minimale Feldmoment bestimmt. Die Spannungen bei den Dehnmessstreifen wurden mit dem Bruttoquerschnitt, diejenigen in der maßgebenden Nietlage mit dem gung einzelner Schnittkräfte unterschiedliche prozentuale Aufteilungen für Innen-/Aussenseite bzw. oberer/ unterer Flansch ergeben, die entsprechend beim Umrechnungsfaktor berücksichtigt wurden.

Eine weitere Besonderheit der Rheinbrücke Eglisau ist die Veränderung des Tragsystems durch den Einbau eines Schottertroges beim Umbau 1982, wodurch einerseits die Längsträger entlastet wurden, anderseits aber der Schottertrog mit seiner grossen Querschnittsfläche Normalkräfte in Brückenlängsrichtung anzieht. Diese Normalkräfte müssen am Brückenende wieder konzentriert, seitlich $\mathrm{zu}$ den Fachwerkpfosten abgetragen werden und verursachen entsprechend zusätzliche Beanspruchungen bei Querträger und Fachwerkpfosten. Zudem wurde nach dem Umbau die Streckenklasse für Bahnverkehr von C3 auf D4 erhöht. Bei der Beurteilung der Ermüdungssicherheit wurden diese Einflüsse berücksichtigt.

\subsection{Dauerfestigkeitsnachweis}

Abgestützt auf die Norm SIA 269/3 [3] wurden für die Hauptfachwerkpfosten sowie für die Längs- und Querträger im Feldbereich die Kerbgruppe 80, für die übrigen maßgebenden Konstruktionsdetails die Kerbgruppe 71 in Rechnung gestellt. In einem ersten Schritt wurde die Dauerfestigkeit untersucht, indem für jede relevante Messstelle der über die gesamte Messperiode maximal gemessene Spannungswechsel unter Berücksichtigung des Umrechnungsfaktors $\mathrm{f}_{\text {tot }}$ in die maßgebende Nietlage im Nachweisquerschnitt umgerechnet und mit der Dauerfestigkeit verglichen wurde (Tabelle 1). Der Dauerfestigkeitsnachweis ist dann erbracht, wenn das Verhältnis $n_{D} \geq 1,0$ beträgt.

Da insbesondere die Längsträger vor dem Umbau 1982 trotz kleinerer Bahnlasten der Streckenklasse C3 höher beansprucht waren, wurde das dreidimensionale Stabmodell an die Situation vor Einbau des Schottertroges angepasst und für die Nutzung bis 1982 ein analoger Dauerfestigkeitsnachweis durchgeführt. Die Beanspruchung im Nachweisquerschnitt wurde dabei am angepassten System mit Bahnlasten der Streckenklasse C3 ermittelt und so die Umrechnungsfaktoren für die Nutzung vor 1982 ermittelt (Tabelle 2). 
Tabelle 1. Ergebnisse des Dauerfestigkeitsnachweises für die maßgebenden Bauteile

Table 1. Results of the verification with respect to the fatigue limit for the determinant structural elements

\begin{tabular}{|c|l|c|c|c|c|c|c|c|c|}
\hline MQS & Erläuterung & $\begin{array}{c}\Delta \sigma_{\mathrm{D}} \\
{\left[\mathrm{N} / \mathrm{mm}^{2}\right]}\end{array}$ & $\begin{array}{c}\max \\
\Delta \sigma_{\mathrm{MQS}} \\
{\left[\mathrm{N} / \mathrm{mm}^{2}\right]}\end{array}$ & $\begin{array}{c}\text { zug. Anz. } \\
\text { Wechsel }\end{array}$ & $\begin{array}{c}\text { DMS } \\
{[-]}\end{array}$ & $\begin{array}{c}\mathrm{f}_{\text {tot }} \\
{[-]}\end{array}$ & $\begin{array}{c}\mathrm{max}_{\mathrm{N}} \\
{\left[\sigma_{\mathrm{NWQS}}\right.} \\
{\left[\mathrm{N} / \mathrm{mm}^{2}\right]}\end{array}$ & $\begin{array}{c}\mathbf{n}_{\mathbf{D}} \\
{[-]}\end{array}$ & $\begin{array}{c}\text { Messung } \\
\text { Monate }\end{array}$ \\
\hline 4 & Pfosten auf Höhe OK QT, QV1 rechts & 58 & 66 & 7 & 21 & 0,71 & 47 & 1,24 & 12 \\
2 & Pfosten auf Höhe UK QT, QV1 links & 58 & 58 & 16 & 37 & 1,12 & 65 & 0,89 & 12 \\
7 & QT, QV1 links & 51 & 22 & 2 & 78 & 1,65 & 36 & 1,42 & 10 \\
8 & QT, QV1 mitte & 58 & 34 & 7 & 76 & 1,18 & 40 & 1,44 & 12 \\
9 & QT, QV1 rechts & 51 & 30 & 75 & 70 & 1,60 & 48 & 1,07 & 11 \\
17 & Längsträger links, Feld 1 bei QV 1 & 51 & 18 & 1 & 58 & 0,64 & 12 & 4,45 & 4 \\
18 & Längsträger Feld 1 Mitte & 58 & 38 & 1 & 64 & 1,43 & 54 & 1,07 & 3 \\
14 & 1. Diagonale links Feld 1 & 51 & 58 & 39 & 13 & 1,71 & 99 & 0,52 & 11 \\
20 & 2. Diagonale rechts Feld 1 & 51 & 62 & 1 & 121 & 1,33 & 82 & 0,62 & 10 \\
25 & Untergurt Feld 10 rechts & 51 & 46 & 10 & 130 & 1,20 & 55 & 0,93 & 11 \\
\hline
\end{tabular}

Tabelle 2. Ergebnisse des Dauerfestigkeitsnachweises für Bahnlasten bis 1982

Table 2. Results of the verification with respect to the fatigue limit for railway loading until 1982

\begin{tabular}{|c|c|c|c|c|c|c|c|c|c|}
\hline MQS & Erläuterung & $\begin{array}{c}\Delta \sigma_{\mathrm{D}} \\
{\left[\mathrm{N} / \mathrm{mm}^{2}\right]}\end{array}$ & $\begin{array}{c}\max \\
\Delta \sigma_{\mathrm{MQS}} \\
{\left[\mathrm{N} / \mathrm{mm}^{2}\right]}\end{array}$ & $\begin{array}{c}\text { zug. Anz. } \\
\text { Wechsel }\end{array}$ & $\begin{array}{c}\text { DMS } \\
{[-]}\end{array}$ & $\begin{array}{l}f_{\text {tot }} \\
{[-]}\end{array}$ & $\begin{array}{c}\max \\
\Delta \sigma_{\mathrm{NWQS}} \\
{\left[\mathrm{N} / \mathrm{mm}^{2}\right]}\end{array}$ & $\begin{array}{l}\mathbf{n}_{\mathbf{D}} \\
{[-]}\end{array}$ & $\begin{array}{l}\text { Messung } \\
\text { Monate }\end{array}$ \\
\hline 4 & Pfosten auf Höhe OK QT, QV1 rechts & 58 & 66 & 7 & 21 & 0,49 & 32 & 1,79 & 12 \\
\hline 2 & Pfosten auf Höhe UK QT, QV1 links & 58 & 58 & 16 & 37 & 0,91 & 53 & 1,10 & 12 \\
\hline 7 & QT, QV1 links & 51 & 22 & 2 & 78 & 1,74 & 38 & 1,34 & 10 \\
\hline 8 & QT, QV1 mitte & 58 & 34 & 7 & 76 & 0,97 & 33 & 1,76 & 12 \\
\hline 9 & QT, QV1 rechts & 51 & 30 & 75 & 70 & 1,69 & 51 & 1,01 & 11 \\
\hline 17 & Längsträger links, Feld 1 bei QV 1 & 51 & 18 & 1 & 58 & 1,26 & 23 & 2,26 & 4 \\
\hline 18 & Längsträger Feld 1 Mitte & 58 & 38 & 1 & 64 & 1,73 & 66 & 0,88 & 3 \\
\hline 14 & 1. Diagonale links Feld 1 & 51 & 58 & 39 & 13 & 1,48 & 86 & 0,60 & 11 \\
\hline 20 & 2. Diagonale rechts Feld 1 & 51 & 62 & 1 & 121 & 1,18 & 73 & 0,71 & 10 \\
\hline 25 & Untergurt Feld 10 rechts & 51 & 46 & 10 & 130 & 1,07 & 49 & 1,04 & 11 \\
\hline
\end{tabular}

Ein Vergleich zeigt auf, dass, wie erwartet, die Längsträger vor dem Einbau des Schottertroges höher beansprucht waren und hierfür keine ausreichende Dauerfestigkeit nachgewiesen werden kann. Aus diesem Grund ist mit Ermüdungsschädigungen $\mathrm{zu}$ rechnen, welche mit der Schadensakkumulationstheorie nach PalmgrenMiner zu berücksichtigen sind.

\subsection{Betriebsfestigkeitsnachweis}

Abgestützt auf die Resultate der Dauerfestigkeitsnachweise für die Nutzungsperioden vor und nach dem Umbau 1982 wurden für Nachweisquerschnitte mit ungenügender Dauerfestigkeit, d.h. für die Pfosten UK Querträger, die 1. und 2. Diagonalen, den Untergurt in Feldmitte sowie für die Längsträger im Randfeld Betriebsfestigkeitsnachweise mittels Schadensakkumulationsberechnung nach Palmgren-Miner durchgeführt. Basis für die

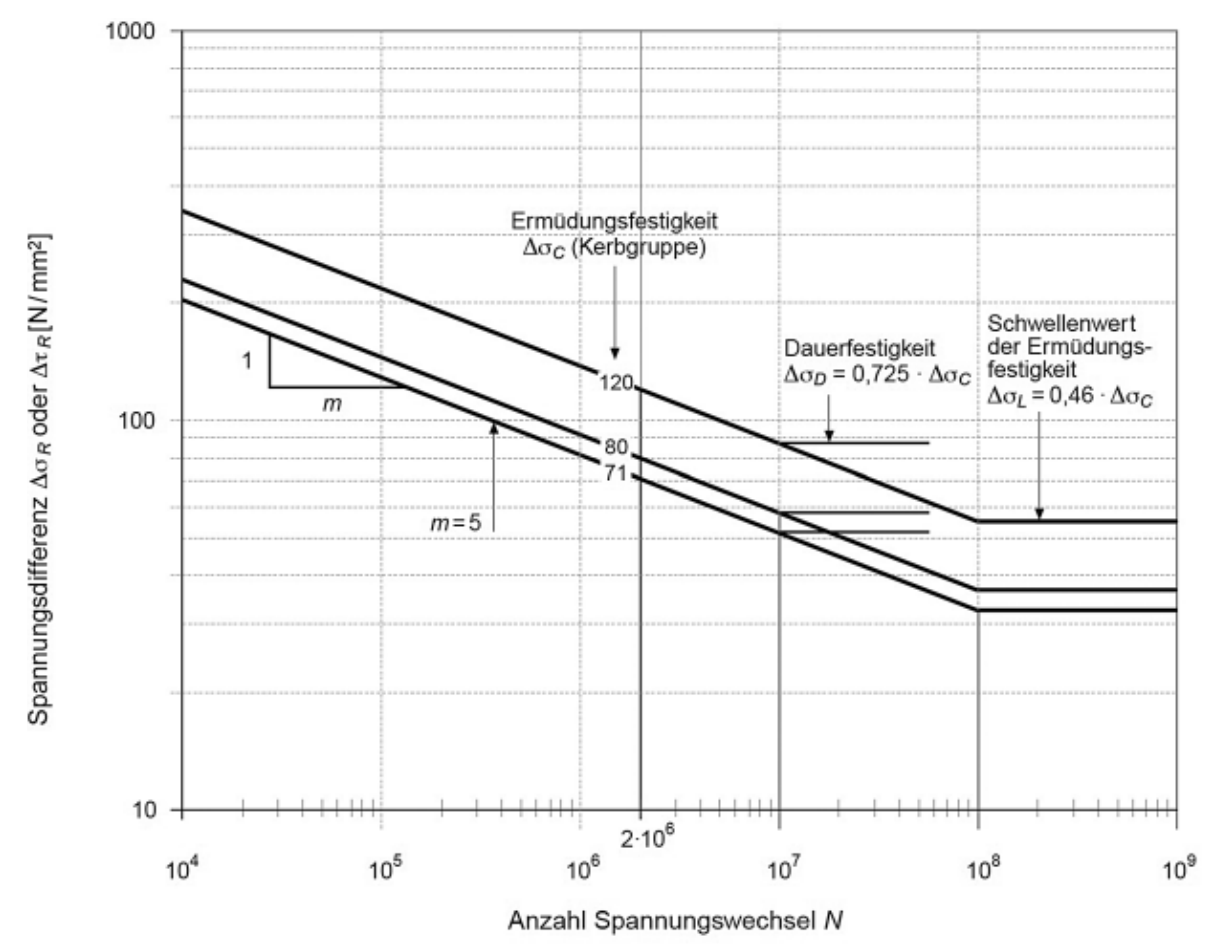

Bild 8. Wöhlerkurven für genietete Konstruktionsdetails gemäß [3]

Fig. 8. Wöhler curves for riveted structural details according to [3] 
Nachweisführung waren die Wöhlerkurven gemäß der Norm SIA 269/3 [3] (Bild 8).

In einem ersten Schritt wurde die Schädigung im Messjahr ermittelt. Dazu wurden die aus den Messwerten aus der Rainflow-Analyse gewonnenen Dehnungs- bzw. Spannungsstufen mittels Umrechnungsfaktor in den Nachweisquerschnitt umgerechnet. Jeder so ermittelten Spannungsstufe wurde nach der Beziehung

$\mathrm{N}_{\mathrm{i}}=\frac{\Delta \sigma_{\mathrm{c}}^{\mathrm{m}} \cdot 2 \cdot 10^{6}}{\Delta \sigma_{\mathrm{i}}^{\mathrm{m}}}$

die maximal mögliche Anzahl Spannungswechsel $\mathrm{N}_{\mathrm{i}}$ zugeordnet. Mit der aus der Rainflow-Analyse pro Spannungsstufe ermittelten Anzahl Spannungswechsel $n_{i}$ konnte so für jede Spannungsstufe und durch Aufsummierung sämtlicher Spannungsstufen des Mehrstufenkollektivs die Schädigung im Messjahr ermittelt werden. Spannungswechsel unterhalb des Schwellenwertes ergeben keine Schädigung (Tabelle 3). Analog konnte auch für die Periode vor der Lasterhöhung und dem Umbau 1982 eine rechnerische Schädigung für das Messjahr ermittelt werden.

Basierend auf statistischen Angaben der Schweizerischen Bundesbahnen zu der Anzahl Zügen und den Gesamtbruttoregistertonnen (GBRT) konnten die bisherigen Verkehrsbelastungen ermittelt werden. Für das Messjahr von April 2011 bis März 2012 resultierte eine Verkehrsbelastung von $23,0 \cdot 10^{6}$ GBRT.

Das zukünftige Verkehrsvolumen beruht auf folgende Prognosen:

für 2025: $33,8 \cdot 10^{6} \mathrm{GBRT} / \mathrm{Jahr}$

für 2035: $38,9 \cdot 10^{6} \mathrm{GBRT} / \mathrm{Jahr}$

(Prognose $2025+15 \%$ )

Dazwischen wird eine lineare $\mathrm{Zu}$ nahme, ab 2035 eine konstante Verkehrsbelastung angenommen. Zusammengefasst resultieren die in der Tabelle 4 festgehaltenen Verkehrsbelastungen.

Basierend auf der ermittelten Ermüdungsschädigung pro Messjahr und der zugeordneten Verkehrsbelastung von 23,0 $\cdot 10^{6}$ GBRT und unter der Annahme einer gleichbleibenden Verkehrszusammensetzung konnte anschließend proportional zur Gesamttonnage für einzelne Zeithorizonte der Schädigungsgrad berechnet werden.

Tabelle 3. Akkumulation des Ermüdungsschadens in der 1. Diagonalen Table 3. Fatigue damage accumulation in the first diagonal

\section{MQS $14 \quad 1$. Diagonale links Feld 1}

NW-QS 13

Kerbgruppe $\quad \Delta \sigma_{\mathrm{C}}=71 \mathrm{~N} / \mathrm{mm}^{2}$

Schwellenwert $\Delta \sigma_{\mathrm{L}}=33 \mathrm{~N} / \mathrm{mm}^{2}$

$\begin{array}{lll}\text { Faktor } & \mathrm{f}_{\text {tot }}= & 1,71[-]\end{array}$

\begin{tabular}{|c|c|c|c|c|}
\hline \multicolumn{5}{|c|}{ DMS 13} \\
\hline $\begin{array}{c}\Delta \sigma \\
{\left[\mathrm{N} / \mathrm{mm}^{2}\right]}\end{array}$ & $\begin{array}{c}\mathrm{f}_{\mathrm{tot}} \cdot \Delta \sigma \\
{\left[\mathrm{N} / \mathrm{mm}^{2}\right]}\end{array}$ & $\begin{array}{c}\text { Anz. } \\
n_{i}\end{array}$ & $\begin{array}{c}\text { Anz. } \\
\mathrm{N}_{\mathrm{i}}\end{array}$ & $\begin{array}{c}\mathrm{n} \cdot 10^{-6} \\
{[-]}\end{array}$ \\
\hline 2,0 & 3,4 & $2,05 \mathrm{E}+07$ & $7,74 \mathrm{E}+12$ & \\
\hline 6,0 & 10,3 & $5,20 \mathrm{E}+04$ & $3,19 \mathrm{E}+10$ & \\
\hline 10,0 & 17,1 & $1,73 \mathrm{E}+04$ & $2,48 \mathrm{E}+09$ & \\
\hline 14,0 & 23,9 & $6,91 \mathrm{E}+03$ & $4,61 \mathrm{E}+08$ & \\
\hline 18,0 & 30,8 & $8,82 \mathrm{E}+03$ & $1,31 \mathrm{E}+08$ & \\
\hline 22,0 & 37,6 & $2,31 \mathrm{E}+04$ & $4,81 \mathrm{E}+07$ & 479,58 \\
\hline 26,0 & 44,4 & $1,49 \mathrm{E}+03$ & $2,09 E+07$ & 71,59 \\
\hline 30,0 & 51,3 & $1,65 \mathrm{E}+03$ & $1,02 \mathrm{E}+07$ & 162,21 \\
\hline 34,0 & 58,1 & $7,87 \mathrm{E}+02$ & $5,45 \mathrm{E}+06$ & 144,32 \\
\hline 38,0 & 64,9 & $5,31 \mathrm{E}+02$ & $3,13 \mathrm{E}+06$ & 169,81 \\
\hline 42,0 & 71,8 & $5,28 \mathrm{E}+02$ & $1,90 \mathrm{E}+06$ & 278,50 \\
\hline 46,0 & 78,6 & $9,30 \mathrm{E}+01$ & $1,20 \mathrm{E}+06$ & 77,31 \\
\hline 50,0 & 85,4 & $7,73 \mathrm{E}+02$ & $7,93 \mathrm{E}+05$ & 974,93 \\
\hline 54,0 & 92,3 & $3,56 \mathrm{E}+02$ & $5,40 \mathrm{E}+05$ & 659,73 \\
\hline 58,0 & 99,1 & $3,90 \mathrm{E}+01$ & $3,77 \mathrm{E}+05$ & 103,31 \\
\hline 62,0 & 105,9 & $0,00 \mathrm{E}+00$ & $2,70 \mathrm{E}+05$ & 0,00 \\
\hline 66,0 & 112,8 & $0,00 \mathrm{E}+00$ & $1,98 \mathrm{E}+05$ & 0,00 \\
\hline 70,0 & 119,6 & $0,00 \mathrm{E}+00$ & $1,47 \mathrm{E}+05$ & 0,00 \\
\hline 74,0 & 126,4 & $0,00 \mathrm{E}+00$ & $1,12 \mathrm{E}+05$ & 0,00 \\
\hline 78,0 & 133,3 & $0,00 \mathrm{E}+00$ & $8,58 \mathrm{E}+04$ & 0,00 \\
\hline 82,0 & 140,1 & $0,00 \mathrm{E}+00$ & $6,68 \mathrm{E}+04$ & 0,00 \\
\hline 86,0 & 146,9 & $0,00 \mathrm{E}+00$ & $5,27 \mathrm{E}+04$ & 0,00 \\
\hline 90,0 & 153,8 & $0,00 \mathrm{E}+00$ & $4,20 \mathrm{E}+04$ & 0,00 \\
\hline 94,0 & 160,6 & $0,00 \mathrm{E}+00$ & $3,38 \mathrm{E}+04$ & 0,00 \\
\hline 98,0 & 167,4 & $0,00 \mathrm{E}+00$ & $2,74 \mathrm{E}+04$ & 0,00 \\
\hline & & & & $\Sigma=3121,3$ \\
\hline Monate: & & 11 & & \\
\hline Anteil: & & $92,0 \%$ & & \\
\hline Faktor: & & 1,087 & & \\
\hline Schädigung i & jahr & 0,0034 & & \\
\hline Entspricht & & $23 \cdot 10^{6} \mathrm{GBRT}$ & & \\
\hline Schädigung bi & & 0,033 & & \\
\hline
\end{tabular}

Vorerst wurde die Schädigung bis zum Umbau 1982 für Bahnlasten der Streckenklasse C3 ermittelt. Es zeigte sich, dass das Tragwerk generell und die Längsträger im Besonderen vor dem Umbau lediglich eine geringe Schädigung erlitten hatten. Anschließend wurden die Schadensakkumulationsberechnungen für ausgezeichnete Zeithorizonte durchgeführt (Tabelle 5).

\section{Beurteilung}

Mit den Messwerten aus dem Langzeit-Monitoring und der Umrechnung in die maßgebenden Nietlagen im Nachweisquerschnitt mit Hilfe des dreidimensionalen Stabmodells konnte für die meisten Bauteile der Nachweis der Dauerfestigkeit erbracht werden. Für die übrigen Bauteile wurde ein Nachweis mittels 
Tabelle 4. Angaben zu den Verkehrsbelastungen

Table 4. Traffic loading data

\begin{tabular}{|c|c|c|c|c|c|}
\hline $\begin{array}{c}\text { Periode } \\
{[-]}\end{array}$ & $\begin{array}{c}\text { GBRT/Jahr } \\
{\left[\cdot 10^{6}\right]}\end{array}$ & $\begin{array}{c}\text { GBRT/Periode } \\
{\left[\cdot 10^{6}\right]}\end{array}$ & $\begin{array}{c}\text { Faktor } \\
{[-]}\end{array}$ & $\begin{array}{c}\boldsymbol{\Sigma} \text { C3 } \\
{\left[\cdot \mathbf{1 0}^{\mathbf{6}}\right]}\end{array}$ & $\begin{array}{c}\boldsymbol{\Sigma} \text { 44 } \\
{\left[\cdot \mathbf{1 0}^{\mathbf{6}}\right]}\end{array}$ \\
\hline $1898-1939$ & 4,70 & 197,4 & 0,89 & $\mathbf{1 7 5 , 5}$ & \\
$1940-1981$ & 9,62 & 404,0 & 0,89 & $\mathbf{5 3 4 , 6}$ & \\
\hline $1982-2011$ & 22,62 & 678,6 & 1,00 & & $\mathbf{6 7 8 , 6}$ \\
$2012-2025$ & 29,40 & 411,6 & 1,00 & & $\mathbf{1 0 9 0 , 2}$ \\
$2026-2035$ & 36,40 & 364,0 & 1,00 & & $\mathbf{1 4 5 4 , 2}$ \\
$2036-2045$ & 38,90 & 389,0 & 1,00 & & $\mathbf{1 8 4 3 , 2}$ \\
$2046-2055$ & 38,90 & 389,0 & 1,00 & & $\mathbf{2 2 3 2 , 2}$ \\
$2056-2065$ & 38,90 & 389,0 & 1,00 & & $\mathbf{2 6 2 1 , 2}$ \\
\hline
\end{tabular}

Schadensakkumulationsberechnung nach Palmgren-Miner durchgeführt, bei welcher der Einfluss des Systemwechsels durch den Einbau des Schottertroges im Jahre 1982 sowie der Wechsel der Streckenklasse von C3 auf D4 berücksichtigt wurde. Mit der seitens der Schweizerischen Bundesbahnen prognostizierten zukünftigen Verkehrsbelastung resultiert für die maßgebende 1. Diagonale bis zum Jahr 2065 lediglich eine Schädigung von 0,42 .

Durch den Einbau des Schottertrogs konnten die Längsträger und damit auch deren Anschlüsse an die Querträger entlastet werden. Die Schadensakkumulationsrechnung zeigt auch, dass die Ermüdungsschädigung infolge mittragender Wirkung der Längsträger im Globalsystem vergleichsweise wenig zunehmen wird, da die Längsträger nicht mehr direkt durch die Bahnlasten beansprucht werden. Die Längs-/Querträgeranschlüsse, die vor allem bei offenen Fahrbahnen für die Ermüdung oft das maßgebende Konstruktionsdetail sind, weisen somit auch eine genügende Ermüdungssicherheit auf.

Das Einhalten des erforderlichen Sicherheitsniveaus erfolgt, indem der mittels Schadensakkumulation berechnete Wert einen gewissen Wert

Tabelle 5. Gesamte prognostizierte Ermüdungsschädigung bis 2065

Table 5. Total projected fatigue damage until 2065

\begin{tabular}{|l|c|c|c|}
\hline Bauteil & \multicolumn{3}{|c|}{ Schädigung bis Ende } \\
& 2011 & 2035 & 2065 \\
\hline Pfosten (UK QT) & 0,005 & 0,011 & 0,019 \\
Längsträger (Feldmitte) & 0,049 & 0,060 & 0,077 \\
1, Diagonale & 0,133 & 0,247 & 0,420 \\
2, Diagonale & 0,025 & 0,045 & 0,075 \\
Untergurt & 0,002 & 0,003 & 0,006 \\
\hline
\end{tabular}

wesentlich intensivere Ermüdungsbeanspruchung.

\section{Folgerungen und Ausblick}

Mit dem aufgezeigten Nachweisverfahren für die Ermüdungssicherheit konnte das primäre Ziel des Monitoring-Projektes „Bahnbrücke Eglisau“, das Tragverhalten und die Ermüdungssicherheit der genieteten Konstruktion auf der Grundlage von messtechnisch erfassten Daten zu beurteilen, erreicht werden. Aufgrund der positiven Erfahrungen mit der Kombination von Tragwerksanalyse und messtechnischer Untersuchung darf erwartet werden, dass bei Anwendung dieser Verfahrensweise zukünftig der Aufwand für Erhaltungsmaßnahmen deutlich reduziert werden kann.

\section{Literatur}

[1] Meyer, C., Bosshard, M., Brühwiler, E.: Nachweis der Ermüdungssicherheit von Brücken - Teil 1: Veranlassung, Ziel und Messkonzept des MonitoringProjekts „Bahnbrücke Eglisau“. Stahlbau 81 (2012), Heft 7, S. 504-509.

[2] Norm SIA 269/1 (2011): Erhaltung von Tragwerken - Einwirkungen. SIA Schweizerischer Ingenieur- und Architektenverein, Zürich.

[3] Norm SIA 269/3 (2011): Erhaltung von Tragwerken - Stahlbau. SIA Schweizerischer Ingenieur- und Architektenverein, Zürich.

\section{Autoren dieses Beitrages:}

Max Bosshard, Dipl. Bauing. ETH, max.bosshard@fbag.ch,

Pirmin Steck, Bauing. MSc. FH,

Flückiger \& Bosshard AG,

Räffelstrasse 32, Postfach 5070, CH-8045 Zürich

Christian Meyer, MSc. Ingenieurgeologie, meyer@terra.ch, terra monitoring ag, Obstgartenstrasse 7, CH-8006 Zürich

Prof. Dr. Eugen Brühwiler,

Dr. és sc. Techn./Dipl. Civil Engineer ETH, eugen.bruehwiler@epfl.ch,

Ecole Polytechnique Fédérale de Lausanne (EPFL),

MCS-ENAC-EPFL, Station 18, CH-1015 Lausanne

Marcel Tschumi, Dr. h. c., Dipl. Bauing. ETH, Rüttistrasse 6, CH-3052 Zollikofen

Senta Haldimann, Dr. és sc. Techn./ Dipl. Bauing. ETH, senta.haldimann@sbb.ch, Schweizerische Bundesbahnen SBB, Infrastruktur, Energie,

Industriestrasse 1, CH-3052 Zollikofen 